\title{
Hemin-mediated neuroglobin induction exerts neuroprotection following ischemic brain injury through PI3K/Akt signaling
}

\author{
BEI ZHANG ${ }^{1}$, XIUPING JI ${ }^{2}$, SHIJUN ZHANG ${ }^{1}$, HUIYUN REN ${ }^{1}$, \\ MINJUAN WANG ${ }^{1}$, CHANGJIANG GUO $^{1}$ and YAJUN LI ${ }^{1}$ \\ Departments of ${ }^{1}$ Neurology and ${ }^{2}$ Traditional Medicine, \\ Affiliated Hospital of Xi'an Medical University, Xi'an, Shaanxi 710077, P.R. China
}

Received February 7, 2013; Accepted June 4, 2013

DOI: $10.3892 / \mathrm{mmr} .2013 .1523$

\begin{abstract}
The aim of the present study was to uncover the mechanism underlying the neuroprotection of Hemin-mediated neuroglobin (Ngb) in an in vivo model of brain injury. Sixty healthy male Sprague-Dawley rats were randomly divided into 5 groups ( $\mathrm{n}=12$, each group): sham surgery, ischemia, Hemin, LY294002 (LY) and Hemin + LY. Focal cerebral ischemia was established by unilateral middle cerebral artery occlusion. Neurological function and cerebral infarction volume were evaluated $24 \mathrm{~h}$ following surgery. Expression of $\mathrm{Ngb}$ and Akt mRNA was detected by RT-PCR, and the expression of Ngb protein and activation of the PI3K/Akt pathway were determined by western blot analysis. No visible damage in the brain and no neurological impairment in the sham surgery group were observed. When compared with the ischemia group, Hemin treatment induced the upregulation of Ngb and Akt mRNA, Ngb protein and phosphorylation of Akt (pAkt). Hemin treatment also improved neurological functions and reduced infarct volume. By contrast, LY treatment increased infarct volume, deteriorated neurological functions and significantly reduced expression of pAkt; however, Ngb mRNA and protein expression was unchanged. When compared with Hemin alone, a combination of Hemin and LY treatment induced more severe brain damage and markedly decreased the expression of pAkt. The results of the present study demonstrated that the PI3K/Akt signaling pathway, which is associated with cell survival, mediates the neuroprotective effects of Hemin-induced Ngb following cerebral ischemia.
\end{abstract}

\section{Introduction}

Neuroglobin $(\mathrm{Ngb})$ is an oxygen-carrying globin and its high oxygen-binding capacity and specific expression in

Correspondence to: Professor Yajun Li, Department of Neurology, Affiliated Hospital of Xi'an Medical University, 48 Fenghao Road, Xi'an, Shaanxi 710077, P.R. China

E-mail: liyajun9@hotmail.com

Key words: PI3K/Akt, neuroglobin, cerebral ischemia the nervous system make it an important candidate in the study of novel mechanisms underlying neural repair following cerebral ischemia and hypoxia (1-3). It has been reported that hypoxia induces Ngb expression (4) and that hypoxia-inducible factor- $1 \alpha$ (HIF- $1 \alpha$ ) positively regulates $\mathrm{Ngb}$ expression (5). In addition, increased expression of $\mathrm{Ngb}$ has been observed in the brain tissue of patients with ischemic stroke (6). Ngb not only enhances cellular survival by regulating cell signaling via $\mathrm{G}$ proteins (7), but also regulates apoptotic mechanisms associated with hypoxia (8-10). A previous study demonstrated that overexpression of $\mathrm{Ngb}$ reduces brain injury induced by intracerebral hemorrhage, providing further evidence that Ngb plays a vital role in neuroprotection (11). In addition, a recent in vivo animal study revealed that exogenous $\mathrm{Ngb}$ protein (fused to the 11-amino-acid human immunodeficiency virus transactivator of transcription protein transduction domain) might be efficiently transduced into neurons in the mouse and protect the brain from mild or moderate ischemic injury (12). The authors reported that $\mathrm{Ngb}$ functions as an endogenous neuroprotective factor in brain ischemia (13).

However, the mechanisms underlying the neuroprotective properties of $\mathrm{Ngb}$ and the relevant signaling pathways have not been fully elucidated. Ngb has been identified to specifically bind to the $G \alpha$ subunit of $G$ proteins, consequently releasing the $G \beta \gamma$ subunit, functioning as a signal transduction factor important for nerve growth factor (NGF)-induced neuroprotection via activation of the PI3K/Akt signaling cascade (14). Of note, transducin, a specific inhibitor of G $\beta \gamma$, prevents NGF-induced neuroprotection, consistent with a role for $\mathrm{G} \beta \gamma$ in a PI3K/Akt-dependant neuroprotection mechanism (15), indicating that Ngb may be involved in this process. HIF-1 $\alpha$ is induced by ischemia and exerts a neuroprotective effect, at least in part, via induction of insulin growth factor, which activates the PI3K/Akt pathway to exert neuroprotection (16). As ischemia and specifically, HIF-1 $\alpha$, directly induce $\mathrm{Ngb}$ expression (5), we hypothesize that the neuroprotective effects of Ngb are mediated by PI3K/Akt signaling. In the present study, the expression of $\mathrm{Ngb}$ and activation of PI3K/Akt was examined in injured brains following focal cerebral ischemia in adult rats and PI3K/Akt signaling was found to be required for the neuroprotective effects of hemin-induced Ngb expression. 


\section{Materials and methods}

Experimental animals and grouping. Healthy adult male Sprague-Dawley rats, weighing $280 \pm 20 \mathrm{~g}(\mathrm{n}=60)$, were provided by the Experimental Animal Center of Xi'an Jiaotong University (Xi'an, China). This study was approved by the Animal Ethics Committee of Xi'an Medical University (Xi'an, China). The rats were randomly divided into five groups ( $\mathrm{n}=12$ /group): i) Sham control; ii) ischemia, with middle cerebral artery occlusion (MCAO); iii) Hemin, which received intraperitoneal injection of $50 \mathrm{mg} / \mathrm{kg}$ Hemin, a specific $\mathrm{Ngb}$ inducer, $1 \mathrm{~h}$ following MCAO; iv) LY, in which tail vein injection of LY294002 (a PI3K/Akt inhibitor, at a concentration of $0.3 \mathrm{mg} / \mathrm{kg}$ ) was performed $15 \mathrm{~min}$ prior to MCAO; v) Hemin + LY, which received LY294002 15 min prior to $\mathrm{MCAO}$ and Hemin $1 \mathrm{hr}$ following MCAO.

Reagents. Rabbit anti-rat polyclonal anti-Ngb antibody (Santa Cruz Biotechnology, Inc., Santa Cruz, CA, USA); rabbit anti-pAkt antibody (Cell Signaling Technology, Inc., Danvers, MA, USA); horseradish peroxidase (HRP)-labeled goat anti-rabbit IgG antibody (Beijing Zhongshan Golden Bridge Biotechnology Co., Ltd., Beijing, China); rabbit anti-rat actin monoclonal antibody (Abcam, Cambridge, UK); RIPA lysis buffer (Bioon, Shanghai, China); enhanced chemiluminescence kit (Santa Cruz Biotechnology, Inc.); BCA protein quantitation kit (Bioon); TRIzol reagent kit (Gibco-BRL, Carlsbad, CA, USA); RT-PCR and PCR amplification kits and DNA ladder (both Takara Bio, Inc., Shiga, Japan); RNA extraction kit (Tiangen Biotech Co., Ltd., Beijing, China); Hemin (Lizhu Biotech Co. Ltd., Shanghai, China); and LY294002 (Santa Cruz Biotechnology, Inc.).

Animal model of MCAO. Rats were anesthetized in a supine position by intraperitoneal injection of $10 \%$ hydrochloride $(0.3 \mathrm{mg} / \mathrm{kg})$. The right middle cerebral artery was permanently occluded as described previously, with minor modifications (17). Briefly, the neck skin of the rat was shaved and sterilized, and an incision was made in the midline of the cervical skin. Next, the right common carotid artery (CCA), external carotid artery (ECA) and internal carotid artery (ICA) were exposed following muscle dissection, and the ECA and CCA were ligated proximally. A small incision was made at the bifurcation of the CCA and a 4-0 monofilament with heated blunt round end $(0.3 \mathrm{~mm}$, sterilized by alcohol and soaked with heparin) was introduced $\sim 18-20 \mathrm{~cm}$ into the origin of the middle cerebral artery via the right ICA, followed by tight ligation of the monofilament around the CCA. Sham surgery was performed by exposing the carotid artery without occlusion of blood flow.

Neurological assessment. Evaluation of neurological function was performed $24 \mathrm{~h}$ prior to and following the induction of ischemia and scored on a 4-point scale as described previously (17). Scale: 0 , normal; 1 , incomplete stretching of left forepaw; 2, circle to the left; 3 , left limbs limping while walking; 4, cannot walk, impaired. Rats exhibiting any of these markers prior to surgery were excluded from the study and rats were then randomly redistributed between groups. Scores from 1-4 were regarded as successful establishment of the MCAO model. Unconscious rats or animals with a score of 0 or mortality within $24 \mathrm{~h}$ were discarded and additional surgeries were performed to reach appropriate numbers of MCAO-positive animals for experiments.

Measurement of volume of cerebral infarction. Brains were dissected $24 \mathrm{~h}$ following surgery ( $\mathrm{n}=6 / \mathrm{group}$ ) and a total of 6 sections were cut with an interval of $2 \mathrm{~mm}$ from Bregma +4.0 to $-8.0 \mathrm{~mm}$, excluding the olfactory bulb, cerebrum and low brain stem. The sections were then stained with a $1 \%$ 2,3,5-triphenyltetrazolium chloride (TTC) solution ( $\mathrm{pH}$ 7.4) and incubated for $30 \mathrm{~min}$ at $37^{\circ} \mathrm{C}$. The infarct area appeared pale on a background of red 'normal' brain. The sections were fixed with a $4 \%$ paraformaldehyde solution. The total volume of each hemisphere and infarction was determined by integration of the distance of the 6 sections. The infarct volume in cubic millimeters was calculated with the following formula: $\mathrm{V}($ infarct volume $)=\mathrm{t} x(\mathrm{~A} 1+\mathrm{A} 2+\ldots \mathrm{An})$, where $\mathrm{t}$ represents the thickness of brain sections and A represents infarction area.

RT-PCR analysis of mRNA expression of $N g b$ and Akt. Brains were dissected $24 \mathrm{~h}$ following surgery ( $\mathrm{n}=6 /$ group) and were immediately stored in liquid nitrogen. mRNA expression of $\mathrm{Ngb}$ and Akt was tested in the sham surgery, ischemia and Hemin groups. Total RNA was extracted according to the TRIzol manufacturer's instructions and RNA was reverse transcribed according to the manufacturer's instructions (Takara, Otsu, Japan). The primers used for RT-PCR were designed using Primer 5.0 software and synthesized by Sangon Biotech Co. Ltd., Shanghai, China: Ngb forward, 5'-AGCCG CAGCCCTCTGGAACA-3' and reverse, 5'-GCAG CATCAATCACAAGCA-3' (176 bp); Akt forward, 5'-CTGG CCAGGCCCAAGCACCG-3' and reverse, 5'-CGT TCACTGTCCACACACTC-3' (109 bp); and GAPDH (internal control) forward, 5'-ACCACCATGGAGAAGGCTGG-3' and reverse, 5'-CTCAGTGTAGCCCAGGATGC-3' (528 bp). PCR conditions were as follows: $94^{\circ} \mathrm{C}$ for $2 \mathrm{~min}, 94^{\circ} \mathrm{C}$ for $1 \mathrm{~min}$, $55^{\circ} \mathrm{C}$ for $1 \mathrm{~min}$ and $72^{\circ} \mathrm{C}$ for $1.5 \mathrm{~min}$, total cycles were 28 and extension was at $72^{\circ} \mathrm{C}$ for $7 \mathrm{~min}$. PCR amplification yield materials were visualized by $1.5 \%$ agarose gel electrophoresis. A UV gel imaging system (Alphalmager2200; Alpha Innotech Corporation, Santa Clara, CA, USA) was used for recording results of electrophoresis. Image J software (NIH, Bethesda, $\mathrm{MD}, \mathrm{USA}$ ) was used for quantitative analysis.

Western blot analysis of $\mathrm{Ngb}$ expression and activation of PISK/AKT. Total protein was extracted from dissected brain tissue and concentration was determined with a BCA protein assay kit. Proteins were separated by $20 \%$ SDS-PAGE gel electrophoresis and transferred onto a PVDF membrane, then blocked at $4^{\circ} \mathrm{C}$ overnight. The membrane was incubated with primary antibodies (rabbit anti-rat polyclonal anti-Ngb antibody (1:500) and rabbit anti-pAkt antibody (1:200) at $4^{\circ} \mathrm{C}$ overnight. Following washing, the membrane was incubated with HRP-conjugated goat anti-rabbit IgG antibody ( Ngb, 1:10,000; pAkt, 1:2,000) and chemiluminescence detection was used to quantify protein expression.

Statistical analysis. Data are expressed as the mean $\pm \mathrm{SD}$ and analyzed by t-test analysis with SPSS 17.0 software (SPSS, 
Inc., Chicago, IL, USA). $\mathrm{P}<0.05$ was considered to indicate a statistically significant difference.

\section{Results}

Mortality. No animals died prior to tissue harvest in the sham control group, one rat died in the ischemia group, two in the LY group, one in the Hemin group (due to acute pulmonary edema following damage to the vagus nerve during surgery) and one in the Hemin + LY group. No significant difference in mortality was found among groups. Animals that died prior to tissue harvest were excluded from further analyses.

Neurological assessment. No neurological deficit was found in the sham control group (score=0). Other groups presented with neurological impairment to various extents $24 \mathrm{~h}$ following surgery. The score in the Hemin group was significantly lower than that in the ischemia group (Table I; $\mathrm{P}<0.05)$. By contrast, the score in the LY group was higher than that in the ischemia group $(\mathrm{P}<0.05)$, indicating that inhibition of the PI3K/Akt pathway increases the severity of neurological impairment. However, no significant difference was found between the Hemin + LY and the ischemia group (Table I), indicating that PI3K/Akt signaling is required for Hemin-mediated neuroprotection.

Treatment with Hemin but not LY294002 inhibits increased infarct volume induced by ischemia. Since measurement of infarct volume more accurately reflects damage caused by cerebral brain ischemia compared with assessment of neurological functions, infarct volume in the ischemic brains of the rats was measured with or without Hemin or LY treatment. Following TCC staining, no pale area was observed in the brains from the sham surgery group. However, ischemia induced a clear infarct volume (Fig. 1). Of note, Hemin treatment significantly reduced ischemia-induced infarct volume by $\sim 2$ fold (Fig. 1; $\mathrm{P}<0.01$ ), indicating that Hemin had a protective effect against brain damage. By contrast, when compared with rats with ischemia only, pre-treatment with LY294002 prior to ischemia was found to significantly increase ischemia-induced infarct volume (Fig. 1; $\mathrm{P}<0.01$ ). Consistent with these observations, when compared with the Hemin group, pre-treatment with LY in the ischemic rats treated with Hemin significantly increased infarct volume by $\sim 2$ fold (Fig. $1 ; \mathrm{P}<0.01$ ). These results indicate that inhibition of PI3K/Akt signaling exacerbates ischemia-induced neurological damage. By contrast, when compared with LY alone, rats treated with Hemin and LY revealed a small but significant decrease in infarct volume, indicating that inhibition of PI3K/Akt signaling was incomplete or that Hemin may mediate neuroprotection via additional unknown mechanisms, but not solely through the PI3K/Akt signaling pathway.

Hemin treatment enhances the expression of $\mathrm{Ngb}$ and Akt mRNA. Ngb mRNA expression was low in the sham control group; however, following ischemia, the expression of Ngb mRNA was increased by $64 \%$ compared with sham controls (Fig. 2; P<0.01). By contrast, the expression of Akt mRNA was decreased by $24 \%$ following ischemia (Fig. 2; $\mathrm{P}<0.01)$. Of note, Hemin treatment significantly increased the

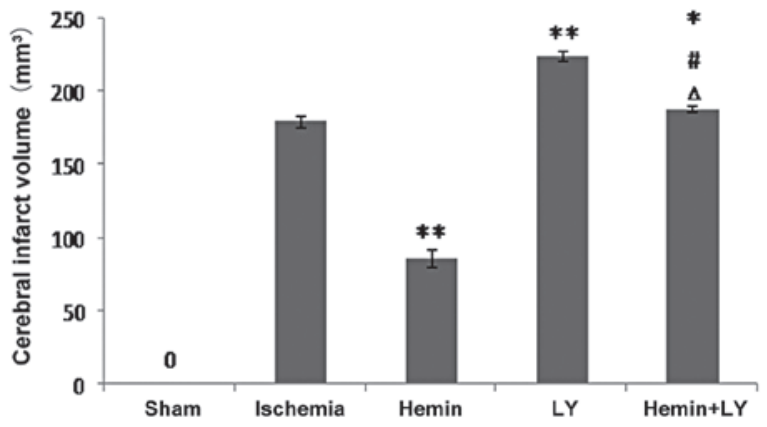

Figure 1. Evaluation of cerebral infarct volume. No infarction was observed in sham controls and the infarct volume was significantly larger with LY and Hemin + LY treatment compared with ischemia alone. A significant reduction of infarct volume was observed with Hemin treatment when compared with ischemia alone $(\mathrm{n}=6)$. ${ }^{*} \mathrm{P}<0.05,{ }^{* *} \mathrm{P}<0.01$ vs. ischemia; ${ }^{\#} \mathrm{P}<0.01$ vs. Hemin; ${ }^{\circ} \mathrm{P}<0.01$, vs. LY. LY, LY294002.

A

Akt

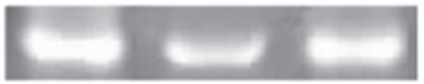

$\mathrm{Ngb}$

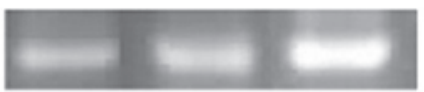

GAPDH

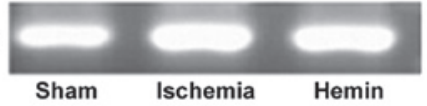

B

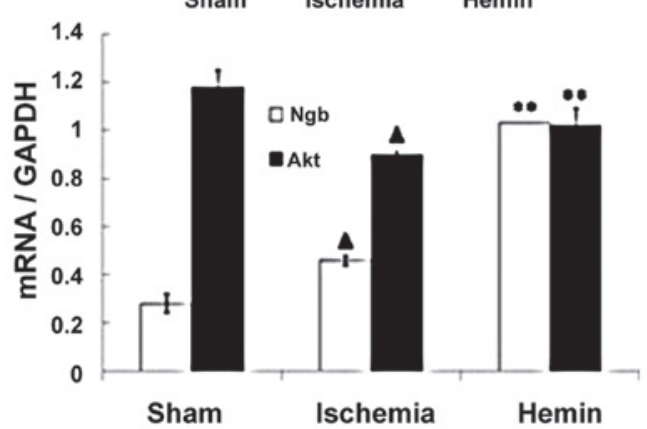

Figure 2. mRNA expression of Ngb and Akt. (A) Band intensity indicates mRNA expression of Ngb, Akt and GAPDH. (B) Quantitative analysis of the expression of $\mathrm{Ngb}$ and Akt mRNA $(\mathrm{n}=6) .{ }^{\wedge} \mathrm{P}<0.01$ vs. sham control; ${ }^{* *} \mathrm{P}<0.01$ vs. ischemia. LY, LY294002; Ngb, neuroglobin.

expression of Ngb and Akt mRNA by 124 and 13\%, respectively (Fig. 2; $\mathrm{P}<0.01$ ).

Expression of $\mathrm{Ng} b$ protein and activation of PI3K/Akt. To examine the expression of Ngb protein and activation of PI3K/Akt, western blot analysis was performed. The expression of $\mathrm{Ngb}$ protein $24 \mathrm{~h}$ following ischemia was increased by $102 \%$ and the levels of pAkt were decreased by $55 \%$ when compared with sham controls. Hemin treatment increased Ngb and pAkt by 47 and 91\%, respectively (Fig. 3; $\mathrm{P}<0.01$ ), whereas LY treatment inhibited pAkt levels but not $\mathrm{Ngb}$ expression (Fig. 3, $\mathrm{P}<0.01$ ). When compared with Hemin treatment, the combination of Hemin and LY treatment inhibited the increased pAkt levels induced by Hemin treatment alone by $50 \%$ (Fig. 3 ; P<0.01). When compared with LY alone, the combination of Hemin and LY treatment induced an increase in pAkt and Ngb expression by 162 and 
Table I. Neurological deficit scores.

\begin{tabular}{lc}
\hline Group & Score \\
\hline Sham control & $0.00 \pm 0.00$ \\
Ischemia & $2.98 \pm 0.76$ \\
Hemin & $2.13 \pm 0.54^{\mathrm{a}}$ \\
LY & $3.42 \pm 1.01^{\mathrm{a}}$ \\
Hemin + LY & $2.87 \pm 0.62^{\mathrm{bc}}$
\end{tabular}

The results are presented as the mean $\pm \mathrm{SD}(\mathrm{n}=12) .{ }^{\mathrm{a}} \mathrm{P}<0.05$ vs. ischemia; ${ }^{\mathrm{b}} \mathrm{P}<0.01$ vs. Hemin; ${ }^{\mathrm{C}} \mathrm{P}<0.01$ vs. LY. LY, LY294002.

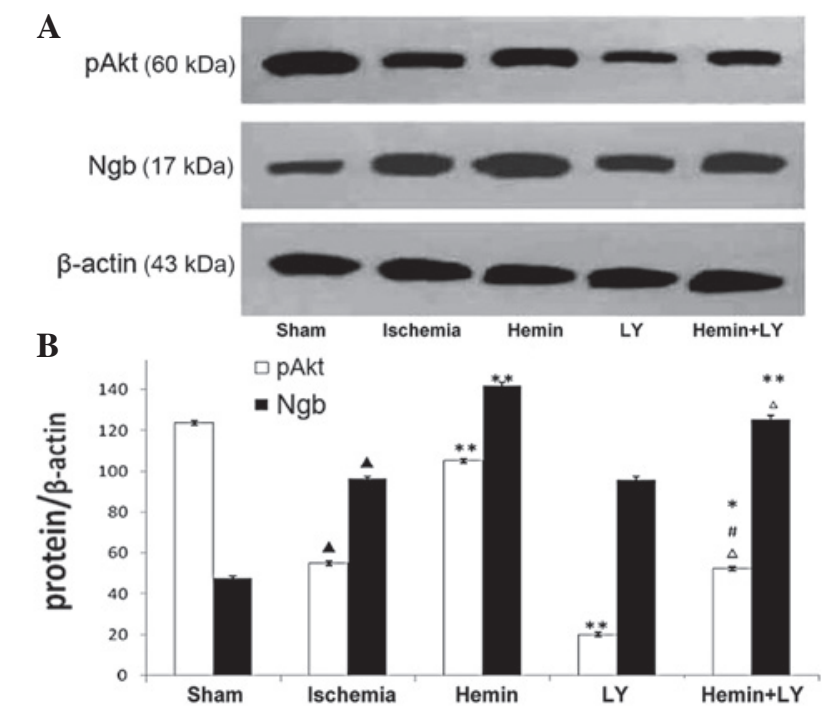

Figure 3. Protein expression of Ngb and pAkt. (A) Gel images reveal Ngb and pAkt levels by western blot analysis. (B) Semi-quantitative analysis of protein expression of $\mathrm{Ngb}$ and pAkt $(\mathrm{n}=6) .{ }^{\wedge} \mathrm{P}<0.01$ vs. sham control; ${ }^{*} \mathrm{P}<0.05$ vs. ischemia; ${ }^{* *} \mathrm{P}<0.01$ vs. ischemia; ${ }^{*} \mathrm{P}<0.01$ vs. Hemin; ${ }^{\wedge} \mathrm{P}<0.01$ vs. $\mathrm{LY}$. $\mathrm{LY}$, LY294002; Ngb, neuroglobin.

31\%, respectively (Fig. 3; $\mathrm{P}<0.01$ ). These results indicate that PI3K/Akt signaling has no effect on Ngb expression, but that Hemin treatment, presumably through $\mathrm{Ngb}$, is able to overcome the ischemia-induced reduction in PI3K/Akt signaling and partially reverses the effects of LY treatment. These results are consistent with our model indicating that $\mathrm{PI} 3 \mathrm{~K} / \mathrm{Akt}$ signaling is a downstream mediator of Ngb neuroprotection.

\section{Discussion}

$\mathrm{Ngb}$ is a globin mainly found in neurons, which is capable of reversibly binding oxygen. It is widely expressed in the vertebrate cerebral cortex, hippocampus, thalamus, hypothalamus and cerebellum (18). Ngb expression in different regions is negatively associated with sensitivity to oxygen. Upregulation of $\mathrm{Ngb}$ expression has been observed to varied extents in response to various pathological conditions, including cerebral ischemia, hypoxia, oxidation and toxicity. Ngb serves as an endogenous neuroprotection factor to enhance tolerance of brain tissues in response to ischemia and hypoxia. It has been revealed that Hemin, a porphyrin containing ferric iron with a chlorine ligand, specifically induces upregulation of Ngb in nerve cells (19). In the present study, intraperitoneal injection of Hemin prior to ischemia enhanced Ngb mRNA expression in rat brains and reversed ischemia-induced neurological impairment and infarction volume. These observations further demonstrated that $\mathrm{Ngb}$ had neuroprotective effects on focal cerebral ischemia and that PI3K/Akt signaling functioned downstream of $\mathrm{Ngb}$ in this pathway.

However, the mechanisms underlying improvement of neurological function and prognosis via endogenous $\mathrm{Ngb}$ remain unclear. Currently, three hypotheses exist: i) $\mathrm{Ngb}$ plays an important physiological role in oxygen absorption, usage and transportation of oxygen to the mitochondria (13); ii) $\mathrm{Ngb}$ protects cells via clearance of reactive oxygen species produced by oxidative stress (20); and iii) Ngb serves as an oxygen sensor to regulate intracellular signal transduction according to changes in oxygen concentration $(7,21)$. It is important to determine which, if any, of these mechanisms are important for $\mathrm{Ngb}$-mediated neuroprotection in future studies.

Signal transduction pathways associated with apoptosis following cerebral ischemia mainly include mitogen-activated protein kinase (MAPK) and PI3K/Akt. It has been hypothesized that MAPK plays a role in regulating apoptosis (22), whereas PI3K/Akt is mainly involved in cellular survival (23). It is known that multiple neurotrophic factors exert neuroprotective effects by inhibiting apoptosis via activation of PI3K/Akt (24). Since it has been previously demonstrated that $\mathrm{PI} 3 \mathrm{~K} / \mathrm{Akt}$ is downregulated in ischemic penumbra within $24 \mathrm{~h}$ of infarction (25), it is likely that PI3K/Akt is important for the processing of neurological damage. Thus, it appears that Ngb activation of PI3K/Akt signaling is a mechanism underlying Ngb-mediated neuroprotection.

The results of the present study indicate that Hemin not only upregulates the expression of Ngb mRNA and protein, but also increases levels of pAkt. In addition, Hemin was found to reduce infarct volume and improve neurological functions. By contrast, administration of the PI3K/Akt-specific inhibitor, LY294002, prior to ischemia increased infarct volume and exacerbated neurological damage, demonstrating the congruence of Ngb and PI3K/Akt function in this system. RT-PCR and western blot analysis revealed that inhibition of PI3K/Akt had no effect on expression of Ngb mRNA and protein, respectively, but it is required for the effects of upregulation of $\mathrm{Ngb}$ expression, indicating that PI3K/Akt functions downstream of Ngb in the signaling pathway associated with neuroprotection. In conclusion, this study delineates a novel mechanism underlying Ngb-mediated neuroprotection, which provides a theoretical basis for the clinical application of $\mathrm{Ngb}$ and also demonstrates that PI3K/Akt signaling may represent a novel target for therapeutic intervention in hypoxic brain injury.

\section{Acknowledgements}

This study was supported by grants from Shaanxi Natural Science Foundation of China (no. 2010JM4054) and research Fund from Shaanxi Provience Education Department (no. 09JK713). The authors would like to thank Medjaden Bioscience Limited for assisting in the preparation of this manuscript. 


\section{References}

1. Kelsen J, Hundahl CA and Hay-Schmidt A: Neuroglobin: endogenous neuroprotectant or maintenance of homeostasis? Stroke 39: e177-e178, 2008.

2. Wang X, Liu J, Zhu H, et al: Effects of neuroglobin overexpression on acute brain injury and long-term outcomes after focal cerebral ischemia. Stroke 39: 1869-1874, 2008.

3. Dong Y, Zhao R, Chen XQ and Yu AC: 14-3-3gamma and neuroglobin are new intrinsic protective factors for cerebral ischemia Mol Neurobiol 41: 218-231, 2010.

4. Yu Z, Liu J, Guo S, et al: Neuroglobin-overexpression alters hypoxic response gene expression in primary neuron culture following oxygen glucose deprivation. Neuroscience 162 : 396-403, 2009

5. Haines B, Demaria M, Mao X, et al: Hypoxia-inducible factor-1 and neuroglobin expression. Neurosci Lett 514: 137-140, 2012

6. Jin K, Mao Y, Mao X, Xie L and Greenberg DA: Neuroglobin expression in ischemic stroke. Stroke 41: 557-559, 2010.

7. Wakasugi K, Nakano T and Morishima I: Oxidized human neuroglobin acts as a heterotrimeric Galpha protein guanine nucleotide dissociation inhibitor. J Biol Chem 278: 36505-36512, 2003.

8. Hota KB, Hota SK, Srivastava RB and Singh SB: Neuroglobin regulates hypoxic response of neuronal cells through Hif- $1 \alpha-$ and Nrf2-mediated mechanism. J Cereb Blood Flow Metab 32: 1046-1060, 2012.

9. Brittain T, Skommer J, Henty K, Birch N and Raychaudhuri S: A role for human neuroglobin in apoptosis. IUBMB Life 62 $878-885,2010$

10. Brittain T and Skommer J: Does a redox cycle provide a mechanism for setting the capacity of neuroglobin to protect cells from apoptosis? IUBMB Life 64: 419-422, 2012.

11. Jin K, Mao X, Xie L and Greenberg DA: Neuroglobin expression in human arteriovenous malformation and intracerebral hemorrhage. Acta Neurochir 111: 315-319, 2011.

12. Cai B, Lin Y, Xue XH,Fang L, Wang N and Wu ZY: TAT-mediated delivery of neuroglobin protects against focal cerebral ischemia in mice. Exp Neurol 227: 224-231, 2011.

13. Sun Y, Jin K, Peel A, Mao XO, Xie L and Greenberg DA: Neuroglobin protects the brain from experimental stroke in vivo. Proc Natl Acad Sci USA 100: 3497-3500, 2003.
14. Dudek H, Datta SR, Franke TF, et al: Regulation of neuronal, survival by the serine-threonine protein kinase Akt. Science 275: 661-665, 1997.

15. Wu EH and Wong YH: Activation of muscarinic M4 receptor augments NGF-induced pro-survival Akt signaling in PC12 cells. Cell Signal 18: 285-293, 2006.

16. Li X, Lu Y, Jin W, Liang K, Mills GB and Fan Z: Autophosphorylation of Akt at threonine 72 and serine 246. A potential mechanism of regulation of Akt kinase activity. J Biol Chem 281: 13837-13843, 2006.

17. Longa EZ, Weinstein PR, Carlson S and Cummins R: Reversible middle cerebral artery occlusion without craniectomy in rats. Stroke 20: 84-91, 1989.

18. Wystub S, Laufs T, Schmidt M, et al: Localization of neuroglobin protein in the mouse brain. Neurosci Lett 346: 114-116, 2003.

19. Zhu Y, Sun Y, Jin K and Greenberg DA: Hemin induces neuroglobin expression in neural cells. Blood 100: 2494-2498, 2002.

20. Jin K, Mao XO, Xie L, Khan AA and Greenberg DA: Neuroglobin protects against nitric oxide toxicity. Neurosci Lett 430: 135-137, 2008.

21. Herold S, Fago A, Weber RE, Dewilde S and Moens L: Reactivity studies of the $\mathrm{Fe}$ (III) and $\mathrm{Fe}(\mathrm{II}) \mathrm{NO}$ forms of human neuroglobin reveal a potential role against oxidative stress. J Biol Chem 279: 22841-22847, 2004.

22. Chaudhry K, Rogers R, Guo M, et al: Matrix metalloproteinase-9 (MMP-9) expression and extracellular signal-regulated kinase 1 and 2 (ERK1/2) activation in exercise-reduced neuronal apoptosis after stroke. Neurosci Lett 474: 109-114, 2010.

23. Jover-Mengual T, Miyawaki T, Latuszek A, Alborch E, Zukin RS and Etgen AM: Acute estradiol protects CA1 neurons from ischemia-induced apoptotic cell death via the PI3K/Akt pathway. Brain Res 1321: 1-12, 2010.

24. Nayak GH, Prentice HM and Milton SL: Neuroprotective signaling pathways are modulated by adenosine in the anoxia tolerant turtle. J Cereb Blood Flow Metab 31: 467-475, 2011.

25. Zhao H, Shimohata T, Wang JQ, et al: Akt contributes to neuroprotection by hypothermia against cerebral ischemia in rats. J Neurosci 25: 9794-9806, 2005. 